\title{
Modelado de producción, consumo y almacenamiento de recursos heterogéneos de un distrito agroindustrial con energías renovables
}

\author{
J. Ramos-Teodoro, F. Rodríguez, M. Castilla, M. Berenguel \\ Departamento de Informática, Universidad de Almería, Ctra. Sacramento s/n, Almería 04120, España \\ Campus de Excelencia Internacional Agroalimentario (ceiA3), CIESOL Centro de Investigación en \\ Energía Solar; \{jeronimo.rt, frrodrig, mcastilla, beren\}@ual.es;
}

\begin{abstract}
Resumen: Este trabajo plantea la aplicación de una metodología de modelado ampliamente utilizada en el ámbito de los «energy hubs» para un distrito agroindustrial ubicado en la provincia de Almería. Debido a que en este se usan recursos renovables de disponibilidad intermitente, se precisan estrategias de gestión eficiente que, mediante técnicas de optimización que emplean modelos simplificados de este tipo, determinen el reparto de recursos entre las diferentes plantas que integran el distrito. Para ello, es necesario implementar un entorno de simulación con el fin de analizar casos concretos, testar nuevos enfoques y desarrollar aplicaciones de ayuda para la toma de decisiones, por lo que se presentan los resultados preliminares del desarrollo de una librería de componentes en Simulink. Asimismo, se ejemplifica el uso del modelo propuesto mediante la resolución de un problema de optimización, en un escenario simplificado, consistente en determinar la cantidad de recursos de entrada y el almacenamiento que se requiere en cada instante de tiempo para minimizar el coste de operación de un invernadero tradicional alimentado por un parking fotovoltaico.
\end{abstract}

Palabras clave: «energy hubs», concentradores de energía, simulación, gestión óptima de recursos, sistemas multi-energía.

\section{Introducción}

Un distrito agroindustrial se puede entender como una entidad socio-territorial caracterizada por la presencia de comunidades de personas y empresas que comparten intereses, y constituida por explotaciones agrícolas, industrias de transformación y de suministro de insumos, e instalaciones de apoyo, localizadas un mismo espacio geográfico [1]. El problema que se aborda en este trabajo se centra en el funcionamiento sinérgico de estos distritos, donde se encuentran sistemas con diferentes objetivos contrapuestos y necesidades de recursos heterogéneos, tanto energéticos (electricidad y calor/frío) como de materiales (agua y $\mathrm{CO}_{2}$ ). En especial, cuando se trata de fuentes renovables, de naturaleza intermitente, es necesario gestionar de forma eficiente el uso de todos los recursos, así como coordinar el flujo entre ellos.

El impulso de la generación distribuida en el sistema eléctrico con el fin de dotar al mismo de flexibilidad, modularidad y eficiencia ha acabado por extenderse a otros sistemas de producción y abastecimiento como las redes de calor y frío o las redes de dióxido de carbono e hidrógeno [2]. La investigación relacionada con conceptos como el de «energy hubs» (concentradores de energía) [3] o sistemas multi-energía [4] pretenden asentar las bases teóricas para tratar agrupaciones de entidades, localizadas en un mismo espacio geográfico, que cuentan con sistemas con objetivos contrapuestos y diferentes necesidades de recursos heterogéneos, tanto energéticos como materiales. Tal es el caso de los distritos agroindustriales, caracterizados 


\section{CONGRESO IBÉRICO DE AGROINENIERÍA \\ X CONGRESSO IBÉRICO DE AGROENGENHARIA \\ 3 - 6 septiembre 2019, Huesca - España}

por la presencia de comunidades de personas y empresas que comparten intereses, y constituida por explotaciones agrícolas, industrias de transformación y de suministro de insumos, e instalaciones de apoyo [5].

En este sentido, es habitual el empleo de modelos simplificados basados en primeros principios para tratar problemas de dimensionado de instalaciones, diseño de las redes distribución o la gestión eficiente de recursos [6]. Aunque existen diversas formulaciones y casos de ejemplo, como se expone en [7], escasean las aplicaciones en distritos agroindustriales, en las que una gestión coordinada de los elementos involucrados puede tener un impacto importante a nivel económico y medioambiental.

En este trabajo se presenta la metodología de modelado, basada en el paradigma empleado en «energy hubs», y los modelos conceptuales y numéricos obtenidos como resultados derivados del mismo. Todo ello particularizado para un distrito productivo agrícola localizado en la provincia de Almería. Finalmente, también se ejemplifica su utilidad en un escenario de simulación del reparto económico de recursos.

El artículo está estructurado de la siguiente forma: en el segundo apartado se describe el conjunto de plantas empleadas y sus principales componentes (para más información se remite a los lectores a la web del proyecto [8]), así como la metodología de modelado aplicada a la misma, basada en balances de masa y energía; en el tercer apartado se presentan los modelos conceptuales y numéricos obtenidos como resultado, junto con su implementación en el entorno MATLAB/Simulink; finalmente, en el cuarto apartado, se resaltan las principales conclusiones y líneas de trabajo para el futuro.

\section{Materiales y métodos}

\subsection{Descripción del distrito agroindustrial}

El distrito agroindustrial que se va a utilizar como ejemplo se enmarca en el proyecto CHROMAE [8] y se compone de los elementos ilustrados en la Figura 1, que a continuación se describen brevemente:

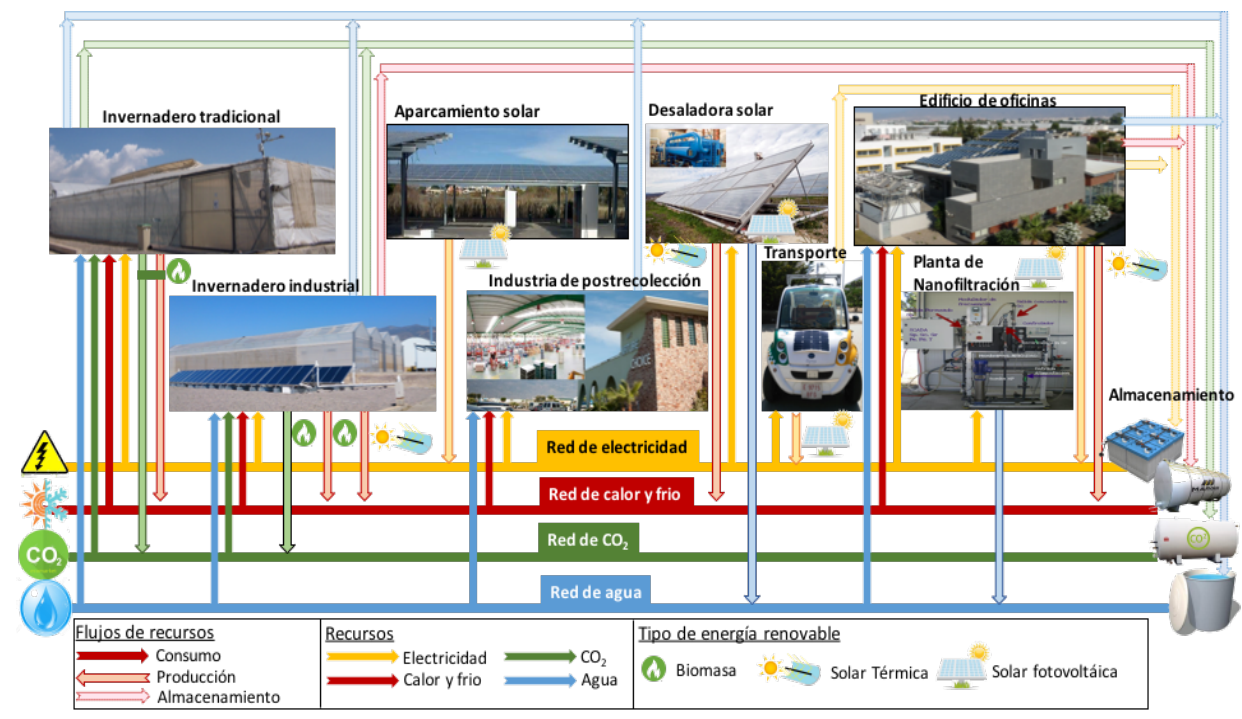

Figura 1. Flujo de recursos entre los diferentes elementos del distrito agroindustrial

- Instalaciones de producción de la Fundación Cajamar. Situadas en la Estación Experimental "Las Palmerillas" en El Ejido (Almería). Se compone de un invernadero tipo parral de $877 \mathrm{~m}^{2}$ con ventilación automatizada y un sistema de deshumidificación de $18 \mathrm{~kW}$. La calefacción es 


\section{CONGRESO IBÉRICO DE AGROINENIERÍA \\ X CONGRESSO IBÉRICO DE AGROENGENHARIA \\ 3-6 septiembre 2019, Huesca - España}

híbrida, constituida por un aerotermo de propano de $95 \mathrm{~kW}$ y una caldera de $174 \mathrm{~kW}$ que emplea biomasa y almacena el $\mathrm{CO}_{2}$ producido en tanques de carbón activo. Dispone de un sistema para el control del aporte de agua y de fertilizantes, así como de su almacenamiento.

- Instalaciones de producción del Instituto de Investigación y Formación Agraria y Pesquera (IFAPA). Situadas en el Centro La Mojonera (Almería), dispone de un invernadero semicerrado industrial de $1000 \mathrm{~m}^{2}$ de altas prestaciones con control de clima dual. La generación térmica está constituida por un campo solar de captadores térmicos planos de gran formato (superficie activa $100 \mathrm{~m}^{2}$ ), un tanque de almacenamiento térmico con capacidad para $7000 \mathrm{~L}$, una caldera de biomasa de $225 \mathrm{~kW}$ con recuperación y almacenamiento de $\mathrm{CO}_{2} \mathrm{y}$ un acumulador de $2000 \mathrm{~L}$.

- Industria de post-recolección y comercialización Hortofrutícola Mabe S.A.T. Ubicada en el término municipal de El Ejido (Almería), dispone de unas instalaciones para el lavado y calibrado de productos hortícolas procedentes de la producción bajo invernadero.

- Edificio de trabajo CIESOL. Se trata de un edificio bioclimático, ubicado en el campus de la Universidad de Almería. Dispone de un sistema de refrigeración y calefacción solar apoyado por fuentes convencionales (con un campo de 80 captadores planos, una máquina de absorción de $70 \mathrm{~kW}$; una torre de refrigeración de $170 \mathrm{~kW}$; dos tanques de almacenamiento de agua caliente de 5000 L cada uno; un calentador auxiliar de $100 \mathrm{~kW}$; dos intercambiadores de placas de 100 $\mathrm{kW}$; dos tanques de agua fría de 2000 L y 3000 L de capacidad); una instalación fotovoltaica (formada por un total de 42 módulos de 60 células solares de una potencia unitaria de $222 \mathrm{Wp}$ ), y dos módulos de baterías de litio-ferrofosfato de 44 Ah y 51,2 V con una capacidad nominal de almacenamiento energético de $4,9 \mathrm{kWh}$ y una potencia de descarga de $5 \mathrm{~kW}$.

- Vehículo eléctrico eCARM. En el campus de la Universidad de Almería se dispone, para tareas de investigación, de un vehículo eléctrico autónomo con un motor DC de $48 \mathrm{~V}(4,3 \mathrm{~kW})$ y un banco de ocho baterías a $6 \mathrm{~V}(210 \mathrm{Ah})$ en serie.

- Aparcamiento solar de la Universidad de Almería. Se trata del aparcamiento del campus, en cuyas marquesinas se ha realizado una instalación fotovoltaica distribuida en trece inversores. La potencia pico total de la instalación es de 1176,48 kW y la potencia nominal de $1015 \mathrm{~kW}$.

- Plantas de desalación solar. Las plantas de desalación solar se encuentran ubicadas en la Plataforma Solar de Almería, en el término municipal de Tabernas. Cuenta con el sistema de desalación AQUASOL, que se compone de una unidad de destilación multi-efecto, un campo de captadores de tipo Concentrador Parabólico Compuesto (CPC), unos tanques de almacenamiento y el apoyo de una caldera de gas pirotubular. La producción de destilado es de $3 \mathrm{~m}^{3} / \mathrm{h}$ para una alimentación nominal de $8 \mathrm{~m}^{3} / \mathrm{h}$. También se trabajará con la plataforma de ensayos para módulos de destilación por membranas, compuesta por un campo solar de captadores planos ( 10 colectores de $2 \mathrm{~m}^{2}$ de superficie cada uno), un tanque de almacenamiento térmico $\left(1,5 \mathrm{~m}^{3}\right)$ y un sistema de distribución.

- Planta de nanofiltración. Ubicada en la Plataforma Solar de Almería, consiste en un sistema de tres membranas cuyo objetivo será retener compuestos orgánicos, micro-contaminantes y otras sustancias que contenga el agua residual para su posterior uso como agua de riego e industrial. Para su funcionamiento es necesario mantener un caudal en unas condiciones óptimas de presión que proporciona una bomba centrífuga de $4 \mathrm{~kW}$. La producción en esta planta se puede realizar de forma continua con un caudal de agua reutilizable de unos $60 \mathrm{~L} / \mathrm{h}$.

\subsection{Modelado de concentradores de energía}

Un enfoque que se adapta a este sistema es el de los concentradores de energía, «energy hubs» [3], término utilizado para designar sistemas que producen, convierten y almacenan diferentes tipos de energía y recursos, sirviendo como interfaz entre productores, consumidores y la propia infraestructura de transporte. La clave de este enfoque reside en su capacidad para modelar sistemas de diversa naturaleza y su aplicación a problemas como diseño y dimensionado de sistemas, planificación de recursos o diseño de redes de distribución [6]. 
En términos generales, los modelos de concentradores de energía se obtienen a partir de balances de masa y energía entre diferentes flujos de recursos de entrada y salida. Estos flujos se representan matemáticamente a través de variables que constituyen los respectivos elementos de los vectores de entrada y salida (Figura 2).

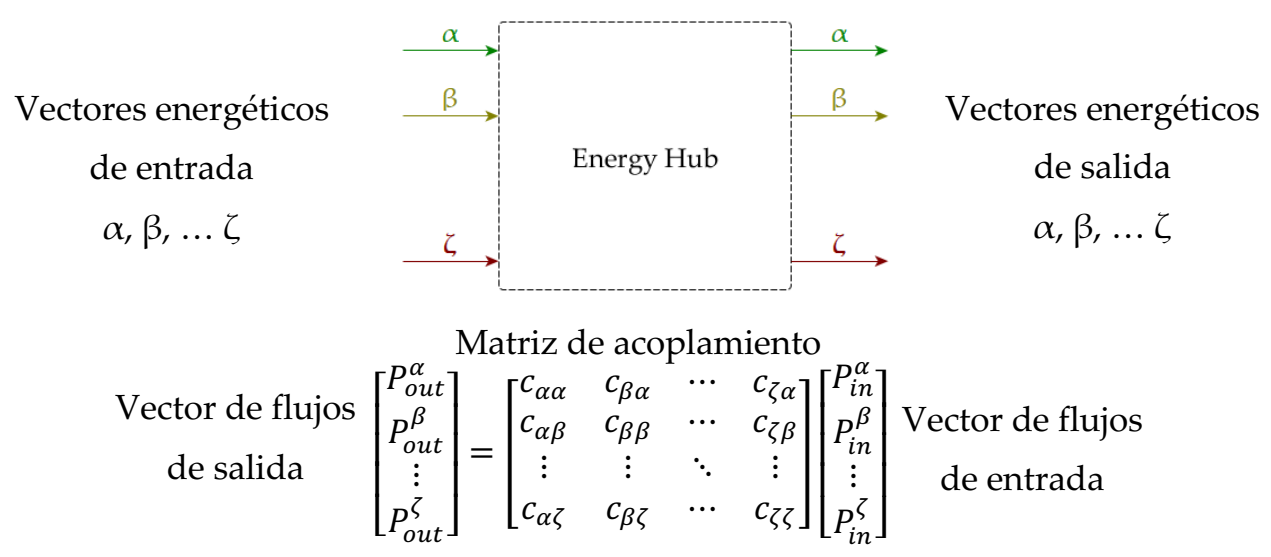

Figura 2. Concepción del modelo genérico de un «energy hub». Fuente: [3]

Las primeras formulaciones [6] definieron la llamada matriz de acoplamiento para establecer la relación entre ellas. Generalmente, se requieren términos adicionales cuando aparecen elementos de almacenamiento, por lo que existe una distinción entre la conversión y el modelo de almacenamiento [9]. Cuando se asume que los dispositivos están lo suficientemente cercanos las pérdidas de recursos solo ocurren en los procesos de conversión y almacenamiento. Para formular un modelo lineal, debe evitarse el uso de coeficientes de reparto; así pues, algunos autores sugirieron introducir tantas variables como dispositivos de conversión, reorganizar el vector de entrada y la matriz de acoplamiento en una nueva ecuación que los relaciona con el vector de salida [10]. Al igual que en [9], este trabajo se considerará al sistema en estado estacionario, en tiempo discreto y con pérdidas exclusivamente en los procesos de almacenamiento y conversión. Los vectores de entrada y salida están formados por las variables que representan la cantidad de energía o recursos intercambiados en cada intervalo de tiempo por el «energy hub», y la matriz de acoplamiento (cuyas dimensiones dependen de éstos).

\section{Resultados y discusión}

\subsection{Modelo de conversión y almacenamiento}

La mayoría de las publicaciones revisadas emplean una formulación lineal del problema por razones de computación. Así lo han hecho también los autores de este trabajo en trabajos previos, por lo que se basándose en el modelo empleado [11], que se expone a continuación, se realizarán adaptaciones sobre este para considerar la inclusión de las redes de distribución en el desempeño del distrito. Considerando que cada uno de los elementos mencionados en el apartado anterior constituye un concentrador de energía, según la definición de los mismos [3], en la Figura 3 se identifican los ocho elementos que componen el distrito (h1-h8) y las características estructurales de cada uno (entradas, salidas y dispositivos).

En cualquiera de ellos, se ha de cumplir el balance de recursos establecido por las ecuaciones (1)-(3) en cada instante de muestreo, definiendo el tiempo de muestreo como el lapso entre dos instantes consecutivos: $T=t(k+1)-t(k)$. La primera expresa que el flujo representado por cada elemento del vector de entrada $\boldsymbol{I}(k)$ ha de ser la suma de todos los flujos posibles asociados a una entrada y contenidos en el vector de recorridos $\boldsymbol{P}(k)$, para lo que se define una matriz de coeficientes constantes (ceros y unos) $\boldsymbol{C}_{\boldsymbol{i}}$. 


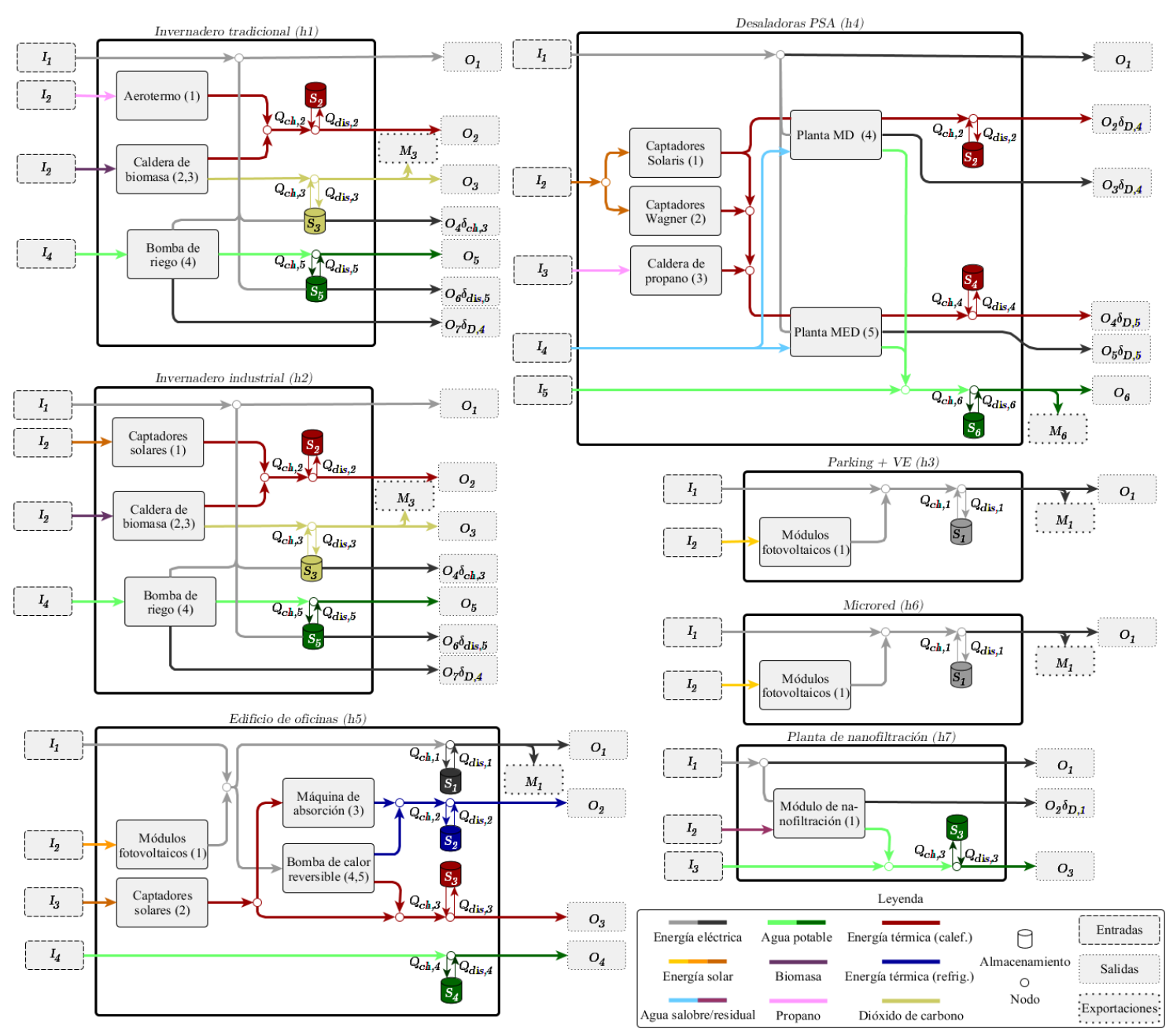

Figura 3. Diagrama de los componentes del distrito basado en el modelo de conversión y almacenamiento empleado

$$
\boldsymbol{I}(k)=\boldsymbol{C}_{\boldsymbol{i}} \boldsymbol{P}(k)
$$

La segunda ecuación relaciona, a través de $\boldsymbol{P}(k)$, los flujos de entrada con respecto a los flujos de salida en forma de demanda propia del concentrador de energía $\boldsymbol{O}(k)$ y en forma de exportaciones al exterior $\boldsymbol{M}(k)$, y con respecto a los flujos de carga $\boldsymbol{Q}_{\boldsymbol{c h}}(k)$ y descarga $\boldsymbol{Q}_{\boldsymbol{d i s}}(k)$ de los dispositivos de almacenamiento. Nótese la aparición de las matrices de acoplamiento $\boldsymbol{C}(k)$, cuyos elementos representan la eficiencia en la conversión de recursos de los diferentes dispositivos de producción, y la matriz diagonal de activación de salidas $\boldsymbol{\delta}_{\boldsymbol{o}}(k)$, cuya diagonal se compone de variables binarias para aquellas demandas de recursos que solo se producen en determinadas circunstancias (por ejemplo, la activación de un sistema de bombeo auxiliar) y de unos para el resto de las salidas.

$$
\boldsymbol{\delta}_{\boldsymbol{o}}(k) \boldsymbol{O}(k)+\boldsymbol{M}(k)=\boldsymbol{C}(k) \boldsymbol{P}(k)-\boldsymbol{Q}_{\boldsymbol{c h}}(k)+\boldsymbol{Q}_{\boldsymbol{d i s}}(k)
$$

La tercera ecuación se emplea para definir los flujos de entrada a cada dispositivo de conversión $\boldsymbol{D}(k)$ por medio de una matriz de acoplamiento $\boldsymbol{C}_{\boldsymbol{d}}(k)$ cuyos elementos, al igual que los de $\boldsymbol{C}(k)$, dependen del recorrido del flujo y de la eficiencia de cada equipo.

$$
\boldsymbol{D}(k)=\boldsymbol{C}_{\boldsymbol{d}}(k) \boldsymbol{P}(k)
$$

Las ecuaciones anteriores se complementan con la ecuación (4) empleada para reflejar la dinámica de los sistemas de almacenamiento, relacionando el estado de carga $\boldsymbol{S}(k)$ de los mismos con los flujos de carga y descarga, teniendo en cuenta la eficiencia en ambos procesos $\left(\boldsymbol{C}_{\boldsymbol{c h}}(k), \boldsymbol{C}_{\boldsymbol{d i s}}(k)\right)$ y la degradación de los recursos almacenados $(\boldsymbol{L}(k))$. 


\section{CONGRESO IBÉRICO DE AGROINENIERÍA}

\section{CONGRESSO IBÉRICO DE AGROENGENHARIA}

3-6 septiembre 2019, Huesca - España

Nótese la aparición del tiempo de muestreo (expresado en minutos) para relacionar las magnitudes correspondientes a los flujos con magnitudes acumuladas.

$$
\boldsymbol{S}(k+1)=\boldsymbol{L}(k) \boldsymbol{S}(k)+\boldsymbol{C}_{\boldsymbol{c h}}(k) \boldsymbol{Q}_{\boldsymbol{c h}}(k) T / 60-\boldsymbol{C}_{\boldsymbol{d i s}}(k) \boldsymbol{Q}_{\boldsymbol{d i s}}(k) T / 60
$$

Sobre algunos de los vectores anteriores es necesario añadir restricciones a partir de los límites y estado de operación de cada dispositivo, mediante las ecuaciones (5)-(10). Los primeros dependen de las características de los equipos e instalaciones, mientras que para determinar la activación o desactivación de los mismos se definen matrices diagonales de variables binarias $(\delta)$ para los dispositivos de conversión $\left(\boldsymbol{\delta}_{\boldsymbol{D}}\right)$, entradas del sistema $\left(\boldsymbol{\delta}_{\boldsymbol{I}}\right)$, exportaciones $\left(\boldsymbol{\delta}_{\boldsymbol{M}}\right)$, carga $\left(\boldsymbol{\delta}_{\boldsymbol{c}}\right)$ y descarga $\left(\boldsymbol{\delta}_{\text {dis }}\right)$.

$$
\begin{aligned}
& \boldsymbol{\delta}_{\boldsymbol{I}}(k) \boldsymbol{I}^{\boldsymbol{m} \text { in }}(k) \leq \boldsymbol{I}(k) \leq \boldsymbol{\delta}_{\boldsymbol{I}}(k) \boldsymbol{I}^{\text {máx }}(k) \quad(5) \quad \boldsymbol{\delta}_{\boldsymbol{c h}}(k) \boldsymbol{Q}_{\boldsymbol{c h}}^{\min }(k) \leq \boldsymbol{Q}_{\boldsymbol{c h}}(k) \leq \boldsymbol{\delta}_{\boldsymbol{c h}}(k) \boldsymbol{Q}_{c \boldsymbol{c h}}^{\text {máx }}(k) \\
& \boldsymbol{\delta}_{\boldsymbol{M}}(k) \boldsymbol{M}^{\boldsymbol{m}^{\mathbf{m} n}}(k) \leq \boldsymbol{M}(k) \leq \boldsymbol{\delta}_{\boldsymbol{M}}(k) \boldsymbol{M}^{\text {máx }}(k) \quad \text { (6) } \quad \boldsymbol{\delta}_{\text {dis }}(k) \boldsymbol{Q}_{\text {dis }}^{\text {min }}(k) \leq \boldsymbol{Q}_{\text {dis }}(k) \leq \boldsymbol{\delta}_{\text {dis }}(k) \boldsymbol{Q}_{\text {dis }}^{\text {máx }}(k) \\
& \boldsymbol{\delta}_{\boldsymbol{D}}(k) \boldsymbol{D}^{\text {minn }}(k) \leq \boldsymbol{D}(k) \leq \boldsymbol{\delta}_{\boldsymbol{D}}(k) \boldsymbol{D}^{\text {máx }}(k) \quad(7) \quad \boldsymbol{S}^{\text {minn }}(k) \leq \boldsymbol{S}(k) \leq \boldsymbol{S}^{\text {máx }}
\end{aligned}
$$

Finalmente, se han de considerar restricciones para los flujos simultáneos (debidos a dispositivos con más de un producto de salida), imponiendo que la suma de variables correspondientes a flujos $(p)$ de un mismo dispositivo sea igual para cada flujo, mediante la ecuación (11); así como restricciones para procesos no simultáneos, como la carga y descarga de dispositivos de almacenamiento, mediante la ecuación (12); el funcionamiento de dispositivos $(d)$ de conversión incompatibles como una bomba de calor reversible, mediante la ecuación (13); y la importación en forma de entradas $(i)$ y exportación en forma de salidas $(o)$ de recursos utilizando un mismo medio, mediante la ecuación (14). Una explicación más detallada de estas restricciones puede encontrarse en [11].

$$
\begin{gathered}
P_{p^{(1)}}(k)+\cdots+P_{p^{(n)}}=P_{p^{(n+1)}}(k)+\cdots+P_{p^{(m)}} \\
\delta_{D, d^{(1)}}(k)+\cdots+\delta_{D, d^{(n)}}(k) \leq 1
\end{gathered}
$$

$$
\begin{gathered}
\delta_{I, i}(k)+\delta_{M, o}(k) \leq 1 \\
\delta_{c h, o}(k)+\delta_{d i s, o}(k) \leq 1
\end{gathered}
$$

\subsection{Modelo de distribución de recursos en sistemas interconectados}

El modelo descrito en el apartado anterior, particularizado para un «energy hub», puede emplearse para representar un conjunto de ellos que compartan recursos a través de diferentes redes de distribución, si se realizan algunas modificaciones. En primer lugar, las ecuaciones (1)(14) ya no solo serían función del instante de muestreo $k$, sino que habrían de cumplirse para los $h$ sistemas considerados. Además de esto, en las ecuaciones (1)-(2) se introducirían variables adicionales para tener en cuenta los recursos importados $\boldsymbol{N}_{\boldsymbol{h} \prime-\boldsymbol{h}}(k, h)$ y exportados $\boldsymbol{N}_{\boldsymbol{h}-\boldsymbol{h}^{\prime}}(k, h)$ dentro de la red, resultando las ecuaciones (15)-(16), respectivamente.

$$
\begin{gathered}
\boldsymbol{I}(k, h)+\sum \boldsymbol{N}_{\boldsymbol{h} \prime \boldsymbol{h}}(k, h)=\boldsymbol{C}_{\boldsymbol{i}} \boldsymbol{P}(k, h) \quad \forall h, h^{\prime}: h^{\prime} \neq h \\
\boldsymbol{\delta}_{\boldsymbol{O}}(k, h) \boldsymbol{O}(k, h)+\boldsymbol{M}(k, h)+\sum \boldsymbol{N}_{\boldsymbol{h}-\boldsymbol{h}^{\prime}}(k, h) \\
=\boldsymbol{C}(k, h) \boldsymbol{P}(k, h)-\boldsymbol{Q}_{\boldsymbol{c h}}(k, h)+\boldsymbol{Q}_{\boldsymbol{d i s}}(k, h) \quad \forall h, h^{\prime}: h^{\prime} \neq h
\end{gathered}
$$

Asimismo, es necesario introducir límites para las nuevas variables junto a variables binarias en forma de matrices diagonales, mediante las ecuaciones (17)-(18), así como impedir que puedan existir flujos bidireccionales, mediante ecuación (19), en la $\mathbf{1}$ es la matriz identidad, y considerar las pérdidas o eficiencia en la transmisión a través de una matriz de acoplamiento $\left(\boldsymbol{C}_{\boldsymbol{N}}\left(k, h, h^{\prime}\right)\right)$. La Figura 3 contempla las variables mencionadas sobre un diagrama ilustrativo de cada planta tratada.

$$
\begin{aligned}
& \boldsymbol{\delta}_{\boldsymbol{h}^{\prime}-\boldsymbol{h}}(k, h) \boldsymbol{N}_{\boldsymbol{h}^{\prime}-\boldsymbol{h}}^{\min }(k, h) \leq \boldsymbol{N}_{\boldsymbol{h}^{\prime}-\boldsymbol{h}}(k, h) \leq \boldsymbol{\delta}_{\boldsymbol{h}^{\prime}-\boldsymbol{h}}(k, h) \boldsymbol{N}_{\boldsymbol{h}^{\prime}-\boldsymbol{h}}^{\max }(k, h) \quad \forall h, h^{\prime}: h^{\prime} \neq h \\
& \boldsymbol{\delta}_{\boldsymbol{h}-\boldsymbol{h}^{\prime}}(k, h) \boldsymbol{N}_{\boldsymbol{h}-\boldsymbol{h}^{\prime}}^{\min }(k, h) \leq \boldsymbol{N}_{\boldsymbol{h}-\boldsymbol{h}^{\prime}}(k, h) \leq \boldsymbol{\delta}_{\boldsymbol{h}-\boldsymbol{h}^{\prime}}(k, h) \boldsymbol{N}_{\boldsymbol{h}-\boldsymbol{h}^{\prime}}^{\operatorname{ma}}(k, h) \quad \forall h, h^{\prime}: h^{\prime} \neq h \\
& \boldsymbol{\delta}_{\boldsymbol{h}-\boldsymbol{h}^{\prime}}(k, h)+\boldsymbol{\delta}_{\boldsymbol{h}^{\prime}-\boldsymbol{h}}(k, h) \leq \mathbf{1} \quad \forall h, h^{\prime}: h^{\prime} \neq h \\
& \boldsymbol{N}_{\boldsymbol{h}-\boldsymbol{h}^{\prime}}(k, h)=\boldsymbol{C}_{\boldsymbol{N}}\left(k, h, h^{\prime}\right) \boldsymbol{N}_{\boldsymbol{h}-\boldsymbol{h}^{\prime}}\left(k, h^{\prime}\right) \quad \forall h, h^{\prime}: h^{\prime} \neq h
\end{aligned}
$$




\section{CONGRESO IBÉRICO DE AGROINENIERÍA}

X CONGRESSO IBÉRICO DE AGROENGENHARIA

3-6 septiembre 2019, Huesca - España

\subsection{Implementación en MATLAB y Simulink: ejemplo de reparto económico de recursos}

Debido a que uno de los objetivos del proyecto CHROMAE es la creación de una librería de componentes de cada uno de los elementos del distrito agroindustrial, en este trabajo se presenta como ejemplo la interfaz utilizada para el aparcamiento fotovoltaico (Figura 4). A la izquierda de la figura 4 se presenta la vista del bloque de Simulink en el editor, mientras que a la derecha aparece desplegado el menú que permite parametrizarlo a partir de sus características físicas (número de paneles conectados en serie y en paralelo, intensidad y voltajes de circuito abierto...). La combinación de diferentes bloques como este permitiría simular el comportamiento de cualquier otro distrito, al igual que añadir nuevos elementos parametrizables.
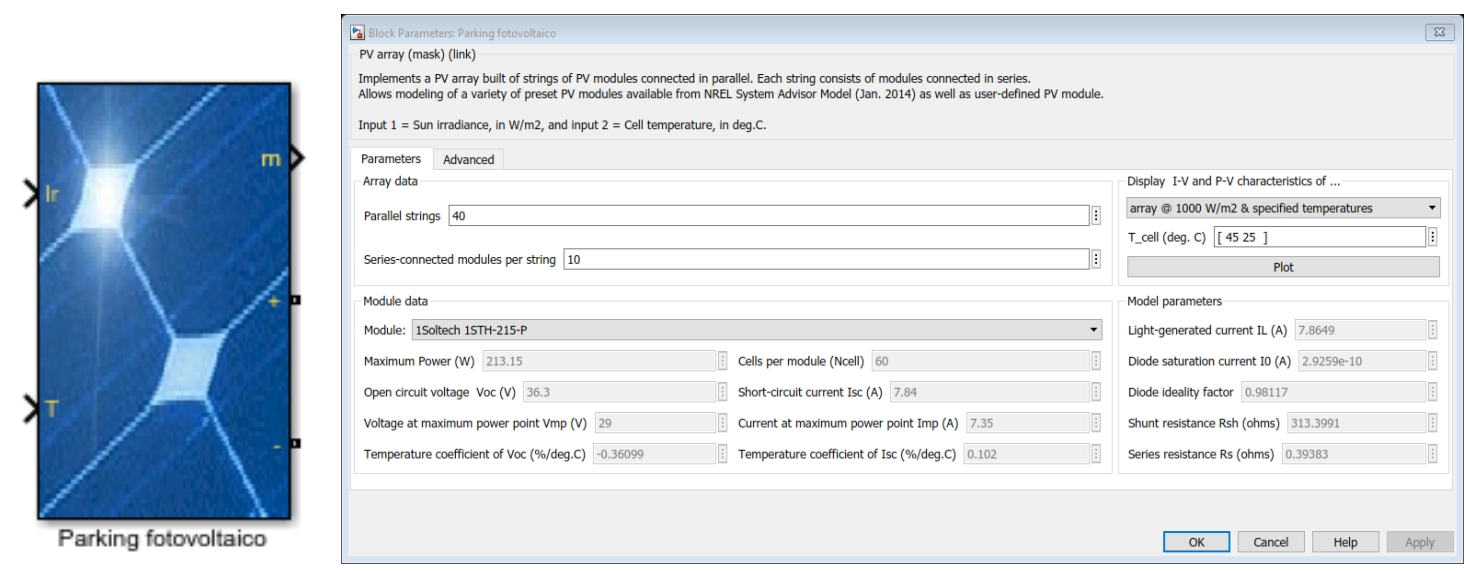

Figura 4. Apariencia de los bloques en Simulink correspondiente al aparcamiento fotovoltaico

Por otro lado, las ecuaciones anteriores pueden constituir las restricciones en un problema de optimización como es el reparto económico de recursos, donde la función objetivo estaría dada por la ecuación (21), siendo $\boldsymbol{c}(k, h)$ el coste de adquisición de cada tipo de recurso en cada sistema y $\boldsymbol{s}(k, h)$ el coste de venta de los recursos producidos. La producción de cada dispositivo, así como el uso de los sistemas de almacenamiento se determinan al resolver el problema y obtener los perfiles de producción que minimizan dicha función.

$$
\begin{gathered}
\min \sum_{k=1}^{H}(\boldsymbol{c}(k, h) \boldsymbol{I}(k, h)-\boldsymbol{s}(k, h) \boldsymbol{M}(k, h)) \\
\text { s.t. ec. (1)-(20) }
\end{gathered}
$$

En la Figura 5 se han incluido los gráficos resultantes (a-f) al ejecutar el algoritmo que se ha desarrollado en código MATLAB para el problema de reparto. Se han empleado datos históricos del invernadero ubicado en la finca experimental «Las Palmerillas», así como, los parámetros característicos de las instalaciones involucradas. Para simplificar, únicamente se ha considerado la interconexión de dos sistemas sin pérdidas en el intercambio de energía: el parking fotovoltaico junto al invernadero tradicional. Por cuestiones prácticas se ha obviado la salida correspondiente a la activación de la bomba de riego para vaciar el depósito de agua, debido a que para el escenario planteado esta no se activa a lo largo del día.

La Figura 5 (a-f) muestra los resultados obtenidos, donde para cada elemento representado se emplea un color asociado al recurso según la leyenda de la Figura 3. Cada figura, organiza la información de la siguiente forma: en ella se hace referencia a la demanda un recurso de salida en cada instante de tiempo, es decir, representan un elemento del vector $\boldsymbol{O}(k)$, y se representan en línea continua gruesa. Para satisfacer dicha demanda, se requieren una cantidad de recursos de entrada (elementos del vector $\boldsymbol{I}(k)$ ), determinada al resolver el problema de optimización, y que se representa en forma de barras apiladas para cada instante de muestreo y para cada recurso. 


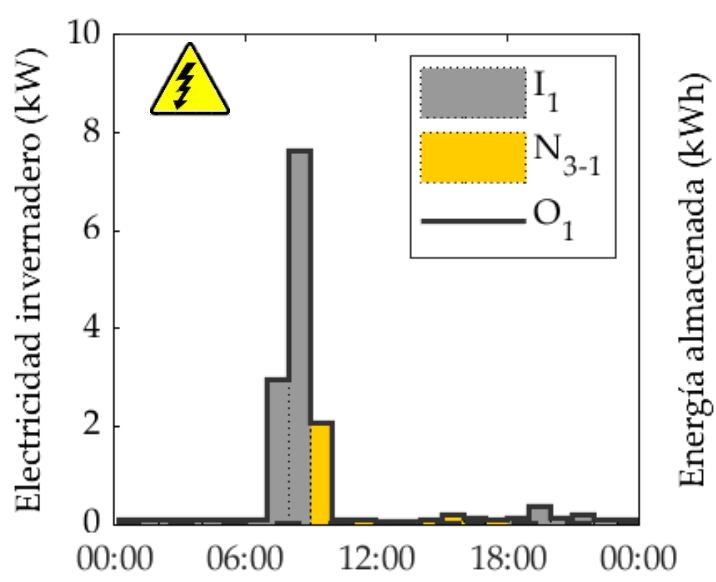

(a)
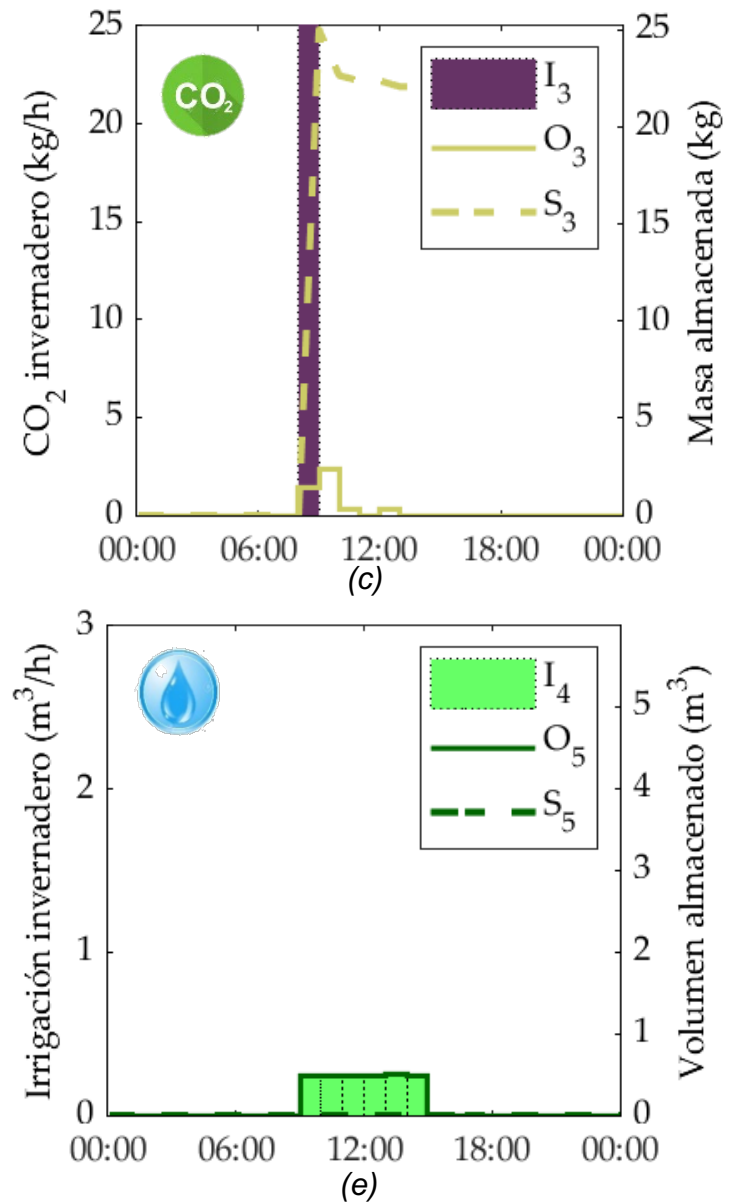
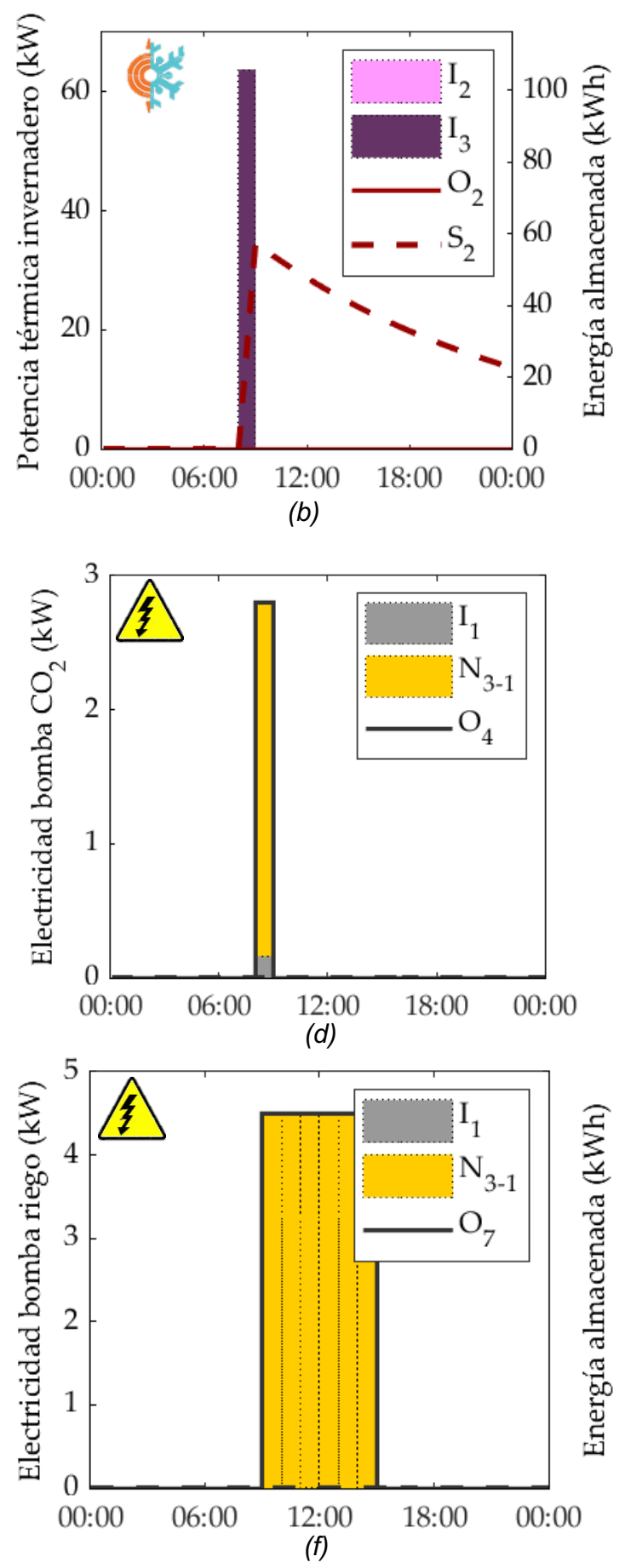

Figura 5. Reparto de recursos entre la planta fotovoltaica y el invernadero el 25/11/2014 (UTC +1)

La diferencia entre el valor de la demanda y la producción a partir de los recursos de entrada (incluyendo el intercambio eléctrico entre los concentradores h1 y h3, $N_{3-1}(k)$ ) determina las operaciones de carga y descarga de los sistemas de almacenamiento, cuya dinámica se representa en línea discontinua: teniendo tendencia creciente durante la carga y decreciente durante la descarga. Finalmente, puede observarse que el aporte solar cubre la demanda eléctrica debida a la activación de la bomba de riego, que está concentrada entre las 9 h y las 15 h del día 24 de noviembre de 2014. Asimismo, se observa que, para cubrir las demandas de $\mathrm{CO}_{2}$, la caldera de biomasa se enciende una única vez durante el día lo cual permite también reducir el número de veces que actúa el sistema de captura de dióxido de carbono. 


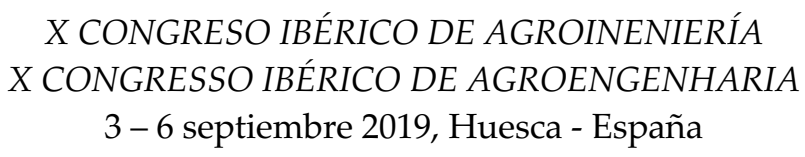

\section{Conclusiones}

Aunque por razones de espacio únicamente se ha limitado a un caso concreto el número de pruebas realizadas, el modelo desarrollado podría hacerse funcionar con conjuntos de datos reales diferentes a los empleados y adaptarlo a cualquier otra estructura de «energy hub». Además, esta formulación del problema contempla la posibilidad de sustituir los submodelos de proceso empleados por otros de mayor o menor complejidad en función de la precisión requerida en los resultados y la capacidad de computación disponible.

Como líneas de investigación futuras, se prevé la adición de nuevos elementos a la librería ya disponible, como resultados del proyecto en el enmarca este trabajo. Por otro lado, se plantean problemas como la gestión eficiente del vehículo eléctrico utilizado en el proyecto, así como la experimentación con sistemas de riego inteligentes, que aprovechen la disponibilidad de recursos para programar el funcionamiento de la bomba del invernadero.

\section{Agradecimientos}

Este trabajo ha sido financiado con el Proyecto I+D+i del Plan Nacional DPI2017-85007-R del Ministerio de Ciencia, Innovación y Universidades y Fondos FEDER.

\section{Referencias}

1. Becattini G. Dal settore industriale al distretto industriale. Rivista di Economia e Politica Industriale. 1979, vol. 1, pp. 1-8.

2. Mukherjee U. et al. Techno-economic, environmental, and safety assessment of hydrogen powered community microgrids; case study in Canada. International Journal of Hydrogen Energy. 2017, vol. 42, n. ${ }^{\circ} 20$, pp. $14333-14349$.

3. Geidl M. et al. Energy Hubs for the Future. IEEE Power and Energy Magazine. 2007, vol. 5, n. ${ }^{\circ}$, pp. 24-30.

4. Mancarella P. MES (multi-energy systems): An overview of concepts and evaluation models. Energy. 2014, vol. 65, pp. 1-17.

5. Caldentey Albert P. El distrito agro-comercial del campo de Dalías (España). Agroalimentaria. 1998, vol. 7, pp. 21-28.

6. Geidl M. y Andersson G. Optimal power flow of multiple energy carriers. IEEE Transactions on Power Systems. 2007, vol. 22, n. ${ }^{\circ}$, pp. 145-155.

7. Mohammadi M., Noorollahi Y., Mohammadi-Ivatloo B., y Yousefi H. Energy hub: From a model to a concept -- A review. Renewable and Sustainable Energy Reviews. 2017, vol. 80, pp. 1512-1527.

8. ARM-TEP197. CHROMAE Project (DPI2017-85007-R). [En línea]. Disponible en: http://www2.ual.es/chromae/. [Accedido: 14-may-2018].

9. Parisio A., Del Vecchio C., y Vaccaro A. A robust optimization approach to energy hub management. International Journal of Electrical Power \& Energy Systems. 2012, vol. 42, n. ${ }^{\circ}$ 1, pp. 98-104.

10. Evins R., Orehounig K., Dorer V., y Carmeliet J. New formulations of the 'energy hub' model to address operational constraints. Energy. 2014, vol. 73, pp. 387-398.

11. Ramos-Teodoro J., Rodríguez F., Berenguel M., y Torres J. L. Heterogeneous resource management in energy hubs with self-consumption: Contributions and application example. Applied Energy. 2018, vol. 229, pp. 537-550. 